\title{
Increasing the bandwidth of a SiGe HBT LNA with Minimum Impact on Noise Figure
}

\author{
Abadahigwa Bimana ${ }^{\dagger}$, SMIEEE, aba.bimana@ieee.org, and \\ Saurabh Sinha ${ }^{\dagger+\dagger}$, SMIEEE, ssinha@ieee.org \\ †Carl \& Emily Fuchs Institute for Microelectronics, Dept. of Electrical, Electronic and Computer \\ Engineering, University of Pretoria, Pretoria, South Africa. \\ $\dagger \dagger$ Faculty of Engineering and the Built Environment, University of Johannesburg, Johannesburg, \\ South Africa.
}

\begin{abstract}
This paper introduces a matching technique for highly sensitive integrated broadband low-noise amplifiers. Noise matching is achieved by the paralleling of identical input transistors. Impedance matching, based on reducing the number of components to the absolute minimum, is done by using the base-collector capacitance as network element. Using a $0.13 \mu \mathrm{m}$ silicon-germanium ( $\mathrm{SiGe}$ ) bipolar complementary metal oxide semiconductor process, simulation results indicate a maximum noise figure of $0.462 \mathrm{~dB}$ at room temperature and a return loss better than $10 \mathrm{~dB}$ from $300 \mathrm{MHz}$ to $1.4 \mathrm{GHz}$. The technique demonstrates that SiGe heterojunction bipolar transistors can be used for cost-effective applications in radio astronomy.
\end{abstract}

Key words: broadband amplifiers; heterojunction bipolar transistors; low-noise amplifiers; noise figure; radio astronomy.

\section{INTRODUCTION}

Advances in radio frequency (RF) integrated circuits have led to the integration of active and passive components on the same semiconductor substrate, departing from technologies from earlier generations that were initially based on discrete devices on printed circuit board and evolved to hybrid circuits, to multichip modules and to monolithic microwave integrated circuits. Despite the benefits of integration, such as high reliability, low cost, low power consumption and small size, the noise performance of RF integrated circuits is impaired by high losses caused by on-chip passive components, substrate losses and interconnection losses. A significant contribution to the noise figure (NF) of integrated low-noise amplifiers (LNA) is made by onchip inductors, owing to their low quality factor.

In a typical LNA architecture, inductors and capacitors are required for input and output matching networks, with the highest degradation of noise performance being caused by the input matching network. While the number of passive components for noise and impedance matching can be low for narrowband topologies, this number may increase significantly for wideband 
LNAs without resistive feedback. Resistive feedback increases the bandwidth but is avoided in sub-1 dB LNA designs, because of its additional thermal noise. For highly sensitive receivers, such as LNAs for radio astronomy, it is desired to reduce the number of passive components at the input to the absolute minimum, especially when the LNA is designed to operate at room temperature.

This paper presents a sub-1dB NF wideband LNA, in the frequency range of $0.3 \mathrm{GHz}$ to 1.4 $\mathrm{GHz}$, at room temperature. The LNA is based on $130 \mathrm{~nm}$ inductively degenerated commonemitter (IDCE) silicon-germanium heterojunction bipolar transistor (SiGe HBT) devices in a cascode configuration. Simultaneous noise and broadband power matching at the input are achieved by only one additional on-chip capacitor, whereas the capacitance of the reverse-biased base-emitter junction at the input transistor is used as circuit element. An output matching network based on a $4^{\text {th }}$ order Butterworth approximation, in conjunction with unconstrained nonlinear optimization, is used to maximize the transfer of power between the output transistor and a $50 \Omega$ load. The values of the elements of the matching network are derived from iterations performed by a MATLAB ${ }^{\mathrm{TM}}$ script resulting in optimum power transfer. The simulation with SpectreRF shows that maximum NF and gain of respectively $0.462 \mathrm{~dB}$ and $18 \mathrm{~dB}$ are achieved in the operating bandwidth.

This paper is structured as follows: Section 2 describes the noise and impedance matching proposed for a narrowband IDCE LNA. Section 3 investigates bandwidth extension techniques. A technique with minimum impact on NF is derived and presented in Section 4. The impact of the bandwidth extension technique on NF is analyzed in Section 5. Simulation results without output matching are presented in Section 6. The proposed output matching network is presented in Section 7. Simulation results of the output matched LNA are presented in Section 8 and the conclusions are found in Section 9.

\section{NOISE AND IMPEDANCE MATCHING TECHNIQUES FOR LNAS}

LNAs have relatively few components. However, their simplicity is misleading, as their design requires several trade-offs. Their performance metrics cannot be optimized simultaneously and complicated concessions are required between NF, bandwidth, gain, power consumption, supply voltage, dynamic range, linearity, stability and matching. In [1], a methodology for achieving 
simultaneous low noise and impedance matching for SiGe HBT based LNAs for the Square Kilometre Array (SKA) is proposed. Owing to the large number of receivers required for the SKA [2], cost-effective LNAs are desired. SiGe HBT based LNAs are therefore low-cost alternatives compared to III-V technologies because of the high $f_{T}$ and $f_{\text {MAX }}$ of SiGe HBTs and their low parasitic resistances [3]. Low noise is obtained by optimal biasing of the input transistor, while noise and impedance matching is achieved through transistor sizing and the IDCE topology. The degenerated common-emitter topology is used in a cascode configuration, which enhances stability performance (through a high reverse isolation $S_{12}$ ) and facilitates input and output impedance matching. The cascode configuration, originating from vacuum tube amplifiers several decades ago, improves performance by decreasing the impact of the Miller's effect to the lower transistor. It also facilitates impedance matching in reducing the coupling between the output and the input. This configuration moreover limits antenna radiations possibly caused by the mixer stage.

The cascode configuration also has disadvantages, including a poor power supply rejection ratio, high output impedance and limited output voltage swing. In addition, the collector current densities of the top and bottom transistors are slightly different owing to the base current of the top transistor and to device mismatches. The LNA is represented in Figure 1 where $Q_{1}$ and $Q_{2}$ are equal in size. Linearity and gain can be improved when $Q_{2}$ is larger than $Q_{1}$, but possibly at the cost of a slight degradation of the NF. A small improvement of the NF can be achieved when $Q_{2}$ is slightly smaller than $Q_{1}$.

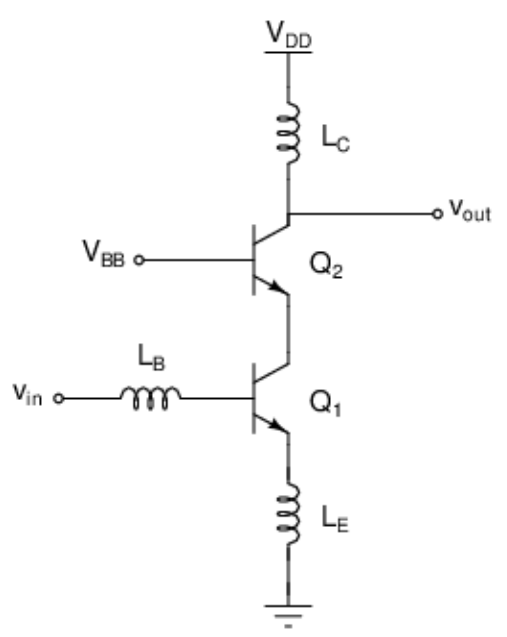

Figure 1 Inductively degenerated common-emitter cascode LNA. 
Noise matching requirements for the circuit in Figure 1 can be analyzed by the classical two-port noise theory. The two-port noise theory, although underutilized in the design of LNAs since its presentation in 1960 [4], allows investigation of how the NF is related to the impedance of the source and the introduction of the concepts of minimum achievable noise factor $F_{\text {min }}$, noise resistance $R_{n}$, optimum source conductance $G_{o p t}$ and susceptance $B_{o p t}$, with $G_{o p t}+j B_{o p t}=Y_{o p t}$, $Y_{\text {opt }}$ being the admittance of the source for which the noise factor is optimum. It can be shown that the noise factor $F$ of a two-port network is given by [5]:

$$
F=F_{\min }+\frac{R_{n}}{G_{s}}\left[\left(G_{s}-G_{o p t}\right)^{2}-\left(B_{s}-B_{o p t}\right)^{2}\right]
$$

where $G_{s}$ and $B_{s}$ are respectively the conductance and the susceptance of the signal source.

The parameters $F_{\min }, R_{n}, G_{\text {opt }}$ and $B_{\text {opt }}$ are known as the four noise parameters and provide, for resistive sources, the source resistance $R_{\text {sopt }}$ for which the minimum NF is achieved, with $R_{\text {sopt }}=R_{e}\left\{1 / G_{\text {opt }}\right\}$. The two-port noise theory allows determination of noise matching conditions, including $R_{n}$, the sensitivity of $F$ to variations of the impedance of the source related to $1 / Y_{\text {opt }}$. Equation (1) shows that, in order to achieve a low $F, F_{\min }$ and $R_{n}$ are required to be low and $Y_{s}$ must be equal or close to $Y_{\text {opt }}$, with $Y_{s}=G_{s}+j B_{s}$.

\section{$2.1 \quad$ Noise Matching}

The performance of receivers is affected by undesired components such as hum, interference and distortion. Moreover, supply sources produce noise and can introduce external noise in the signal path [6]. However, such noise is extrinsic to receivers and, contrary to intrinsic noise, can generally be suppressed. Intrinsic noise in RF amplifiers is random and mainly due to active components, matching networks and the direct current (DC) biasing circuitry. Noise in active components and in biasing circuitry is of thermal, shot, flicker, avalanche and generationrecombination types, while in passive matching networks, the noise is mainly thermal [5]. In active devices, electronic noise is related to their specifications, to temperature, to the bias current and to the operating frequency. For SiGe HBTs, intrinsic noise is closely related to parasitic resistances such as base and emitter resistances $r_{b}$ and $r_{e}$, the unity gain frequency $f_{T}$ and the current gain $\beta_{o}$. It is shown in [7] that, for the common-emitter configuration, $F_{\min }$ for SiGe HBTs is given by: 


$$
F_{\text {min }}=1+\frac{n}{\beta_{o}}+\sqrt{\frac{q I_{C}}{2 k T}\left(r_{b}+r_{e}\right)\left(\frac{f^{2}}{f_{T}^{2}}+\frac{1}{\beta_{o}}\right)+\frac{n^{2}}{\beta_{o}}}
$$

where $I_{C}$ is the collector current, $q$ is the electron charge, $k$ is the Boltzmann constant, $T$ the absolute temperature, $n$ the collector ideality factor and $f$ the operating frequency.

It can also be shown that $F_{\text {min }}$ reaches a minimum for $I_{c}=I_{\text {copt }}$, corresponding to the emitter current density $J_{\text {copt }}$. Furthermore, $F_{\min }$ is invariant to $I_{c}$ as long as $J_{\text {copt }}$ is constant. Hence, for a given technology process, the same minimum value of $F_{\min }$ is achieved by transistors of different emitter length $\left(l_{e}\right)$ and width $\left(w_{e}\right)$ when $J_{\text {copt }}$ is constant.

The noise resistance $R_{n}$ and the source resistance for minimum noise $R_{\text {sopt }}$ are given by the following equations for the common emitter SiGe HBT [7]:

$$
\begin{aligned}
& R_{n} \approx \frac{n^{2} V_{T}}{2 J_{c} w_{e} l_{e}}+\left(r_{b l}+r_{e l}\right) \frac{1}{l_{e}} \\
& R_{\text {sopt }} \approx \frac{R_{n} f_{T}}{f} \frac{\sqrt{\frac{I_{C}}{2 V_{T}}\left(r_{b}+r_{e}\right)\left(1+\frac{f_{T}^{2}}{\beta_{o} f^{2}}\right)+\frac{n^{2} f_{T}^{2}}{4 \beta_{o} f^{2}}}}{\frac{I_{C}}{2 V_{T}}\left(r_{b}+r_{e}\right)\left(1+\frac{f_{T}^{2}}{\beta_{o} f^{2}}\right)+\frac{n^{2}}{4}\left(1+\frac{f_{T}^{2}}{4 \beta_{o} f^{2}}\right)}
\end{aligned}
$$

where $r_{b l}=r_{b} l_{e}$ and $r_{e l}=r_{e} l_{e}$ are technology constants, $n=1$ and $J_{c}$ is the emitter current density.

If $I_{c}$ is replaced by $J_{c} w_{e} l_{e}$, (4) is rewritten as:

$$
R_{\text {sopt }} \approx \frac{R_{n} f_{T}}{f} \frac{\sqrt{\frac{J_{C} w_{e}}{2 V_{T}}\left(r_{b} l_{e}+r_{e} l_{e}\right)\left(1+\frac{f_{T}^{2}}{\beta_{o} f^{2}}\right)+\frac{n^{2} f_{T}^{2}}{4 \beta_{o} f^{2}}}}{\frac{J_{C} w_{e}}{2 V_{T}}\left(r_{b} l_{e}+r_{e} l_{e}\right)\left(1+\frac{f_{T}^{2}}{\beta_{o} f^{2}}\right)+\frac{n^{2}}{4}\left(1+\frac{f_{T}^{2}}{4 \beta_{o} f^{2}}\right)} .
$$

The second factor of (5) is not related to $l_{e}$ for a given emitter width, technology process, current density and operating frequency. Therefore, $R_{\text {sopt }}$ is proportional to $1 / l_{e}$ and noise matching can be achieved by varying the length of the emitter. For submicron technologies, $R_{\text {sopt }}$ can be much higher than the source resistance, which is typically $50 \Omega$ at microwaves and for devices of 
maximum size for the manufacturing technology. In such a case, several transistors can be connected in parallel. It is shown in [8] that the NF is invariant to the paralleling of identical transistors and that $R_{\text {opt }}$ is inversely proportional to the number of transistors in parallel.

\subsection{Impedance Matching}

The input impedance of the amplifier in Figure 1 can be expressed by (6), for frequencies that are much lower than $f_{T}$, for which the impact of the Miller effect on the input transistor can be neglected.

$Z_{\text {in }}=r_{b}+\frac{I_{c} q}{k T C_{\pi}} L_{E}+j \omega\left(L_{B}+L_{E}\right)-\frac{j}{\omega C_{\pi}} \cong r_{b}+\omega_{T} L_{E}+j \omega\left(L_{B}+L_{E}\right)-\frac{j}{\omega C_{\pi}}(6)$

with $\frac{I_{c} q}{k T C_{\pi}}=\frac{g_{m}}{C_{\pi}} \cong \omega_{T}$ where $C_{\pi}$ is the base-emitter capacitance, $g_{m}$ the transconductance, $\omega$ the angular frequency, and $\omega_{T}=2 \pi f_{T}$.

From (6), it is found that the real part of $Z_{\text {in }}$ is independent of frequency and is related to the emitter inductor $L_{E}$, to the transconductance $g_{m}$ and to the base-emitter capacitance $C_{\pi}$. The impedance of the emitter inductor $L_{E}$ is seen as a real resistance when viewed from the input. Hence, this inductor can be used to match the real part of the input impedance to the resistance of the source. The input impedance also has a capacitive reactance, due to the base-emitter capacitance $C_{\pi}$, which must be cancelled by an inductive reactance for resistive sources. The capacitive reactive component is cancelled by $L_{E}$ and by the inductor $L_{B}$ in the base circuit, an inductor that is sized to resonate with $L_{E}$ and $C_{\pi}$ at the operating frequency. Impedance matching is achieved in a narrow frequency band whose bandwidth is determined by the quality factor $Q_{\text {in }}$ at the input. Narrow bandwidth refers to a bandwidth of less than $20 \%$ of the center frequency $[1]$.

In summary, minimum noise is achieved by the biasing of the input transistor at $I_{\text {copt }}$, while noise and power matching are achieved when the source resistance $R_{s}$, when $R_{\text {sopt }}, L_{B}$ and $L_{E}$ are related by the equations [9]:

$$
R_{s} \cong r_{b}+\omega_{T} L_{E}
$$




$$
\begin{aligned}
& L_{B} \cong \frac{1}{\omega^{2} C_{\pi}}-L_{E} \\
& R_{s} \cong R_{\text {sopt }} \\
& \omega L_{B}=X_{\text {opt }}-\omega L_{E}
\end{aligned}
$$

where $X_{\text {opt }}$ is the imaginary part of $1 / Y_{\text {opt }}$.

\section{BANDWIDTH EXTENSION FOR LNAS}

The noise performance of an LNA is strongly related to the noise caused by the input matching network, whereas the impact of the output matching network on noise performance is small and can be negligible, as indicated by Friis's formula [10]. The desired input impedance can be attained by noiseless or noisy input matching networks or by the use of feedback networks. For narrowband applications, simple matching can be achieved by the use of L, T or $\Pi$ networks.

Bandwidth is easily increased by a shunt resistance at the input or by resistive feedback. However, the shunt and feedback resistances increase the NF and reduce the gain. Typically, the $\mathrm{NF}$ increases by more than $6 \mathrm{~dB}$ in the case of resistive shunt while the impairment is smaller for resistive feedback, the resistance used in the resistive feedback generally being high. It may then appear that wideband matching for ultra-low noise amplifiers can only be achieved by complex matching networks and by configurations such as balanced amplifiers, distributed amplifiers and transmission lines. The power consumption of these configurations is high and they entail large areas of the chip. In addition, balanced amplifiers demand large quadrature couplers that increase losses [11]; their noise performance is not better than $3 \mathrm{~dB}$ [12]. Bandwidth increase can also be attained by impedance transformers, by emitter degeneration with resistive and capacitive loading [13] and by input matching networks such as ladder filters [14].

It has been shown that traditional techniques used for bandwidth expansion require additional components at the input, components that impair the noise performance of the LNA. Such techniques include the use of an impedance transformer and of various types of broadband matching networks. As any additional component in the input network affects the NF, it is desirable to reduce the number of matching components to the strict minimum. In addition, to 
limit the impact of bandwidth increase to the NF, resistive components cannot be used in the signal path, through matching or feedback networks.

\section{BANDWIDTH EXTENSION TECHNIQUE}

The input matching of the broadband common-source inductively degenerated LNA in [16] is investigated in [13]. It is found that, for low frequencies, input matching is achieved by the capacitive feedback from the capacitive load in series with a resistor through the gate-drain capacitance. For high frequencies, matching is obtained owing to the inductive feedback in the source circuit. The LNA requires few components at its input and uses the intrinsic gate-drain capacitance coupled to an external capacitor between the drain of the input transistor and the source of the cascode transistor to increase the matching bandwidth. The design strategy constitutes using unfavorable circuit characteristics such as parasitics as circuit elements. This is well paraphrased by Thomas Lee in the preface of [15]: “To no small degree, an important lesson in RF and microwave design is that there are always irreducible parasitics. Rather than conceding defeat, one must exploit them as circuit elements." A detailed analysis of the bandwidth expansion of a SiGe HBT based LNA with minimal impact on noise performance is provided in this section.

A simplified small-signal model of a cascode amplifier is shown in Figure 2, where the parasitic base and emitter resistances are neglected and the input impedance of the cascode transistor is $Z_{L}$. The input impedance has been estimated in (6), where the base-collector capacitance $C_{\mu}=$ $C_{b c}$ has been neglected. Neglecting the base resistance as well, the input impedance at the base is given by the expression:

$$
Z_{\text {in }} \cong \omega_{T} L_{E}+j \omega\left(L_{E}\right)-\frac{j}{\omega C_{b e}}
$$

Taking into account the effect of the base-collector capacitance, the input impedance is equal to

$Z_{i n} \| \frac{1}{Y_{b}}$ where $Y_{b}$ is the admittance seen at the base, after the input capacitor $C_{b e}$ as shown in Figure 2. 


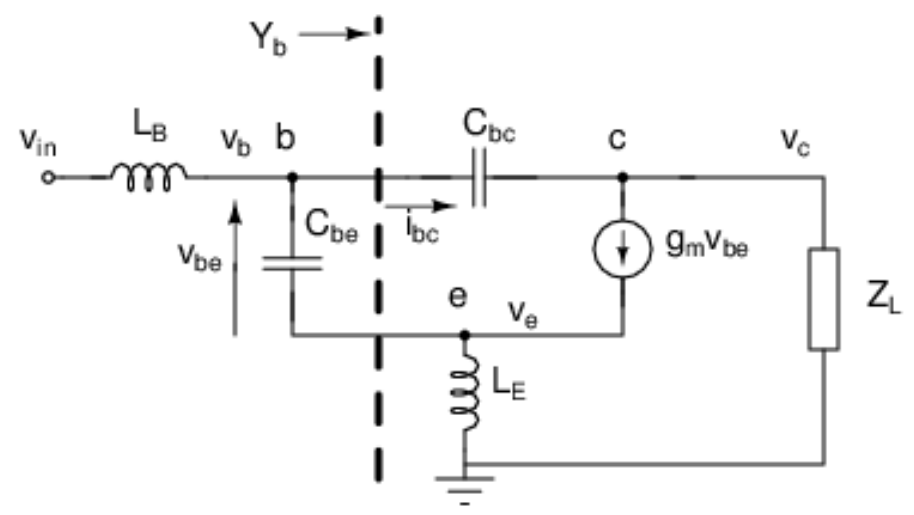

Figure 2 Simplified small-signal model of the IDCE cascode amplifier.

The admittance $Y_{b}$ is calculated by dividing the current $i_{b c}$ by the voltage $v_{b}$ and it can be shown that $Y_{b}$ is given by:

$$
Y_{b}=j \omega C_{\mu}+\frac{j \omega C_{\mu} g_{m} Z_{L}}{1+j \omega L_{E} g_{m}}
$$

Hence, the admittance $Y_{b}$ is given by $Y_{b}=Y_{1} \| Y_{2}$ with $Y_{1}=j \omega C_{\mu}$ and $Y_{2}=\frac{j \omega C_{\mu} g_{m} Z_{L}}{1+j \omega L_{E} g_{m}}$. Therefore $Y_{b}$ is the result of a capacitor $C_{\mu}$ in parallel with the impedance $Z_{2}=1 / Y_{2}$. The impedance $Z_{2}$ is rewritten as:

$$
Z_{2}=\frac{\left(1+j \omega L_{E} g_{m}\right) Y_{L}}{j \omega C_{\mu} g_{m}}
$$

where $Y_{L}=1 / Z_{L}$.

A capacitor of value $C_{L}$ is added between the collector of the input and the emitter of the cascode transistor. $Z_{L}$ is then seen as $r_{o}$ in parallel with $C_{L}$ in series with $1 / g_{m}, 1 / g_{m}$ being the input resistance of the common-base cascode transistor. The transconductances of both the input and cascode transistors are identical. The impedance $Z_{L}$ is represented in Figure 3. 


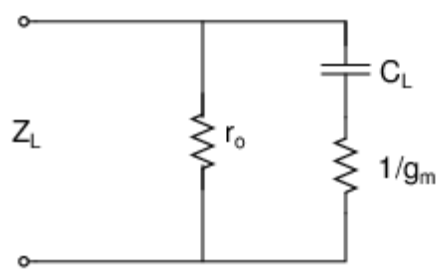

Figure 3 Impedance at the output of the input transistor.

The expression of $Z_{L}$ is given by:

$$
Y_{L}=\frac{1}{r_{o}}+\frac{j \omega g_{m} C_{L}}{g_{m}+j \omega C_{L}}
$$

In replacing $Y_{L}$ by its expression in (14), (13) becomes:

$$
Z_{2}=\frac{1}{j \omega C_{\mu} g_{m} r_{o}}+\frac{L_{E}}{C_{\mu} r_{o}}+\frac{\left(1+g_{m} j \omega L_{E}\right)}{g_{m} j \omega C_{\mu}} \frac{j \omega C_{L} g_{m}}{\left(g_{m}+j \omega C_{L}\right)}
$$

Equation (15) shows that $Z_{2}$ is formed by a capacitor of value $C_{\mu} g_{m} r_{o}$, a resistance of value $\frac{L_{E}}{C_{\mu} r_{o}}$ and an impedance $Z_{3}$ equal to the last term of (15), all in series.

The impedance $Z_{3}$ is given by:

$$
Z_{3}=\frac{C_{L}}{C_{\mu}\left(g_{m}+j \omega C_{L}\right)}+\frac{L_{E} j \omega C_{L} g_{m}}{C_{\mu}\left(g_{m}+j \omega C_{L}\right)}
$$

The impedance $Z_{3}$ is equal to the sum of $Z_{4}$ and $Z_{5}$, given by the relations:

$$
\begin{aligned}
& Z_{4}=\frac{C_{L}}{C_{\mu}\left(g_{m}+j \omega C_{L}\right)} \\
& Z_{5}=\frac{L_{E} j \omega C_{L} g_{m}}{C_{\mu}\left(g_{m}+j \omega C_{L}\right)} .
\end{aligned}
$$


The impedance $Z_{4}$ is equivalent to a resistor of value $\frac{C_{L}}{C_{\mu} g_{m}}$ in parallel of a capacitor of value $C_{\mu}$. The impedance $Z_{5}$ is equivalent to a resistor of value $\frac{L_{E} g_{m}}{C_{\mu}}$ in parallel with an inductance of value $\frac{L_{E} C_{L}}{C_{\mu}}$.

Combining $Z_{\text {in }}$ and the successive impedance transformations of $Z_{L}$, the input impedance of the cascode amplifier is represented in Figure 4.

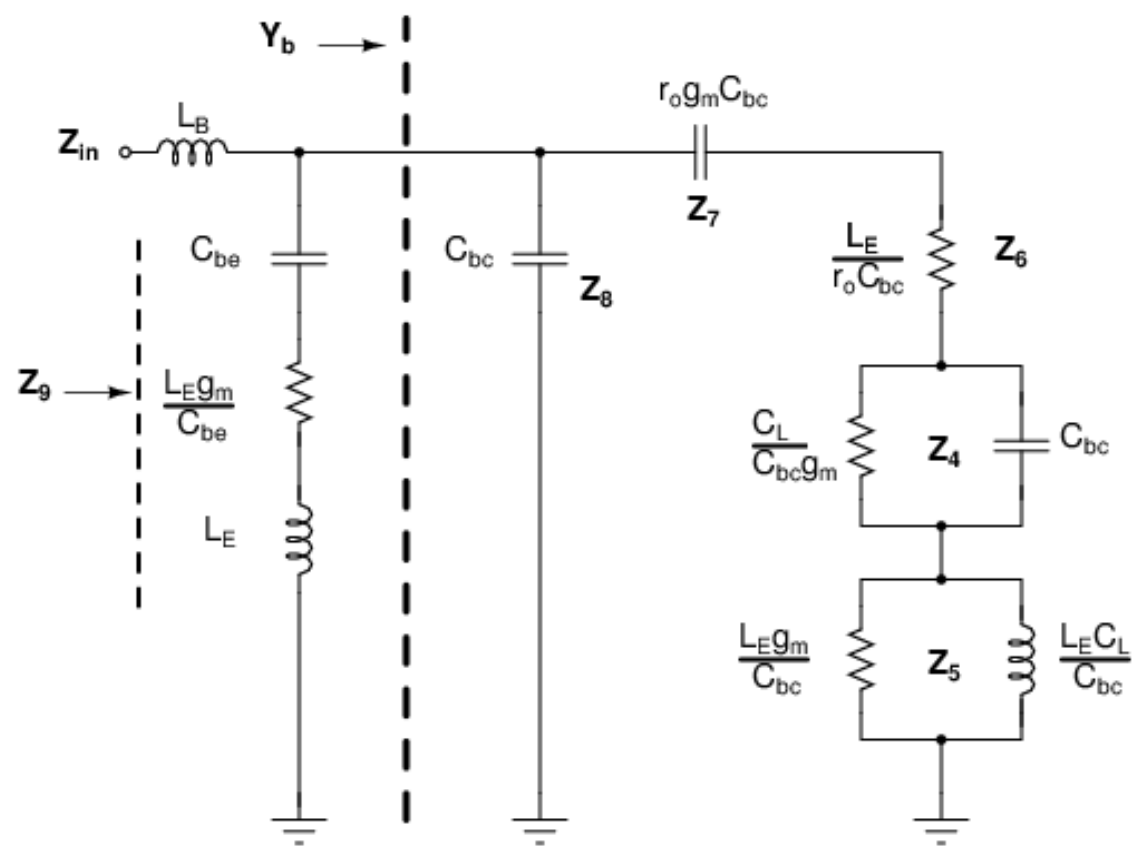

Figure 4 Input impedance of the modifier cascode amplifier.

The circuit in Figure 4 is a double-tuned resonant circuit with the inductance $L_{B}$ in series with the impedance $Z_{9}$, being the first RLC tuned circuit. The resonant frequency $f_{1}$ of this circuit is given by:

$$
f_{1}=\frac{1}{2 \pi \sqrt{\left(L_{B}+L_{E}\right) C_{b e}}}
$$

The order of magnitude of passive components of Figure 4 is evaluated from the typical parameters of a six-finger SiGe HBT. The transistor is biased at a base voltage of $0.867 \mathrm{~V}$ and 
the area of the transistor is $0.12 \times 6 \times 1 \mu \mathrm{m}^{2}$. For an operating frequency of $1 \mathrm{GHz}$, it can be shown that $Z_{4}$ is mainly resistive and that $Z_{5}$ is inductive. Hence, $Z_{7}, Z_{4}$ and $Z_{5}$ form the second tuned RLC circuit. The frequency $f_{2}$ of this tuned circuit can be approximated by:

$$
f_{2}=\frac{1}{2 \pi \sqrt{r_{o} g_{m} L_{E} C_{L}}} .
$$

Resonance at $f_{2}$ is due to capacitive feedback and $f_{2}$ is lower than $f_{1}$, the values of the capacitance of $Z_{7}$ and of the inductance in $Z_{5}$ being higher than $C_{\pi}$ and $L_{B}+L_{E}$. Owing to an adequate determination of the frequencies $f_{1}$ and $f_{2}$, the combined impedance of the coupled tuned circuits can achieve an input reflection coefficient $S_{11}$ that is less than $-10 \mathrm{~dB}$ in a wide band.

\section{IMPACT ON NF}

The second stage of the cascode amplifier being coupled to the first stage through the capacitor $C_{L}$, the question of the impact of this coupling on the NF arises. Assuming that the gain of the input stage is high, the contribution of the second stage $\left(Q_{2}\right)$ to the NF is neglected. The bandwidth-enhanced cascode LNA is represented in Figure 5 as a cascade of two two-port networks formed by $Q_{1}$, the input transistor, and $C_{L}$.

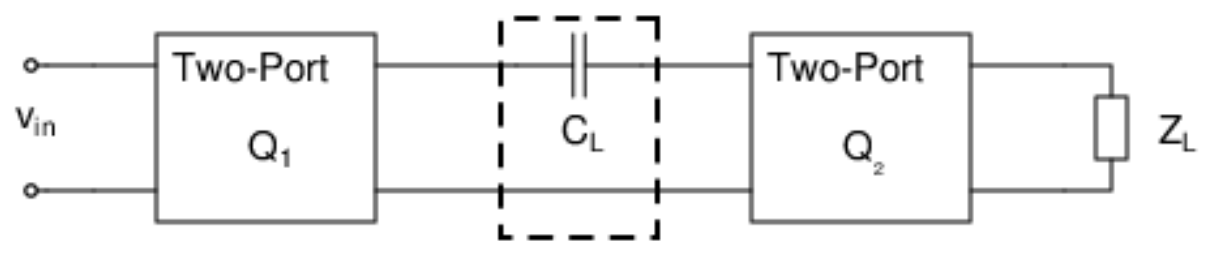

Figure 5 Bandwidth-enhanced cascode LNA.

The NF of the two-port networks in Figure 5 is given by:

$$
F=1+\frac{Z^{+} C_{A} Z}{2 k T \operatorname{Re}\left\{Z_{S}\right\}}
$$


where $C_{A}$ is the noise correlation matrix of the cascaded network resulting from $Q_{1}$ and $C_{L}, Z_{s}$ is the impedance of the source, $Z^{+}$is the Hermitian conjugate of $Z$ with $Z=\left[\begin{array}{c}1 \\ Z_{s}^{*}\end{array}\right]$. The noise correlation matrix $C_{A}$ is expressed as:

$$
C_{A}=A_{1} C_{A 2} A_{1}^{+}+C_{A 1}
$$

where $A_{1}$ is the transmission matrix of the input stage and $C_{A 1}$ is its noise correlation matrix. $C_{A 2}$ is the noise correlation matrix of the two-port network formed by $C_{L}$.

The noise correlation matrix $C_{A 2}$ is nil, as its diagonal terms are real values that represent the power spectrum of each noise source and the off-diagonal terms represent their cross-power spectra; these terms are nil for a noiseless network. The noise correlation matrix of the input stage is given by:

$$
\left.C_{A 1}=2 k T\left[\begin{array}{c}
R_{n} \\
{\left[\frac{F_{\text {min }}-1}{2}-R_{n} Y_{\text {sopt }}\right.}
\end{array}\right] \begin{array}{c}
{\left[\frac{F_{\text {min }}-1}{2}-R_{n} Y_{\text {sopt }}^{*}\right.}
\end{array}\right] .
$$

It is then concluded that $C_{A}$ is not related to $C_{L}$, hence $C_{L}$ does not impair the noise factor of the LNA.

\section{SIMULATION RESULTS}

SiGe HBTs of maximum size $\left(0.12 \times 18 \mu^{2}\right)$ from the $130 \mathrm{~nm}$ IBM BiCMOS8HP process are used for the cascode configuration. $F_{\min }$ and $R_{o p t}$ for the cascode LNA are determined by simulation with SpectreRF. Noise matching is done at the high end of the frequency band since, $F_{\text {min }}$ being an increasing function of frequency, the minimum achievable noise is better than $F_{\text {min }}$ in the bandwidth of the LNA. It is found that $F_{\text {min }}=0.337 \mathrm{~dB}$ and that the base bias voltage $V_{B}$ required for minimum NF is $0.7705 \mathrm{~V}$ at $1.4 \mathrm{GHz}$. The plot of $F_{\min }$ as a function of $V_{B}$ is shown in Figure 6. 


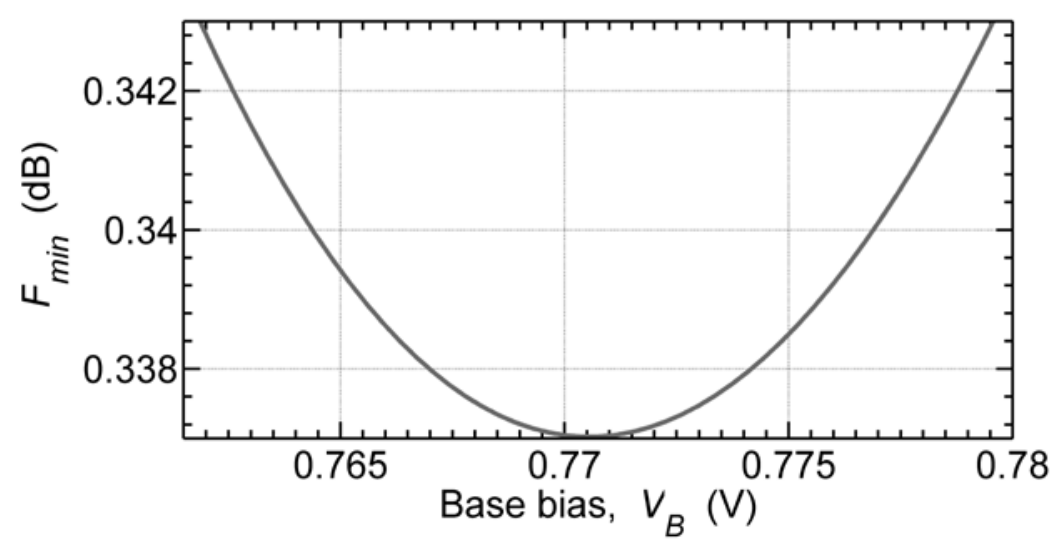

Figure 6 Optimum base bias voltage for the cascode amplifier.

A parametric plot of $R_{o p t}$ as a function of frequency allows one to determine that 14 parallel transistors are required for noise matching at $1.4 \mathrm{GHz} . R_{\text {opt }}$ is chosen to be slightly higher than $50 \Omega$ because of the parasitic resistance of the base inductor $L_{B}$. The impact of transistor paralleling on $F_{\min }$ is negligible, $F_{\min }$ is unchanged when 14 transistors are connected in parallel at the input and at the output. The base inductor is approximated by matching the reactive part $X_{\text {opt }}$ of the noise impedance to $L_{B}$. Hence, at $1.4 \mathrm{GHz}, \mathrm{NF}=F_{\min }$. The emitter inductor is calculated from (7) and then fine-tuned by simulation of $S_{11}$, as shown in Figure 7.

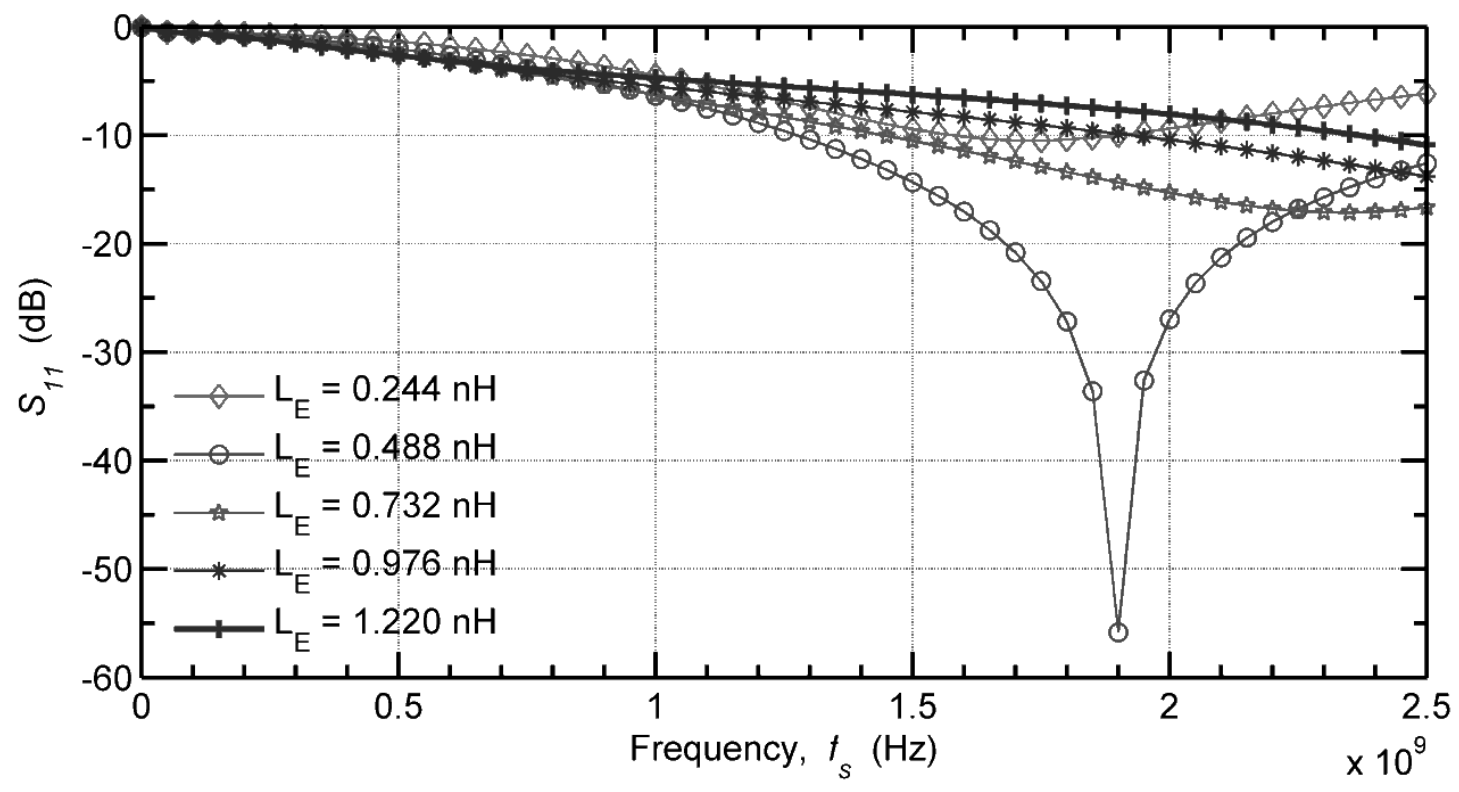

Figure $7 S_{11}$ versus frequency and $L_{E}$. 
As $L_{B}$ has been determined on the basis of noise matching only, the addition of $L_{E}$ cancels the noise matching at $1.4 \mathrm{GHz}$ and the relation between $L_{B}$ and $L_{E}$ is determined by (10). Therefore, the imaginary part of $Z_{\text {in }}$ is not nil at $1.4 \mathrm{GHz}$. A new value of $L_{B}$ is determined by a parametric simulation of NF as a function of frequency and of $L_{B}$. The resonant frequency of the input matching circuit is found by simulation of $S_{11}$ as a function of frequency. It is found that $S_{11}$ is minimal at $1.89 \mathrm{GHz}$, a frequency higher than $1.4 \mathrm{GHz}$ at which noise matching has been performed. Therefore, noise is matched at $1.4 \mathrm{GHz}$ while power is matched at $1.89 \mathrm{GHz}$, which improves bandwidth extension. The latter frequency corresponds to $f_{1}$, as predicted in Section 4 and given by (19).

Narrowband matching being achieved, a capacitor $C_{L}$ is introduced between the collector of the input transistor and the emitter of the cascode transistor. The value of $C_{L}$ is found by seeking a flat response for $S_{11}$ in the operating bandwidth.

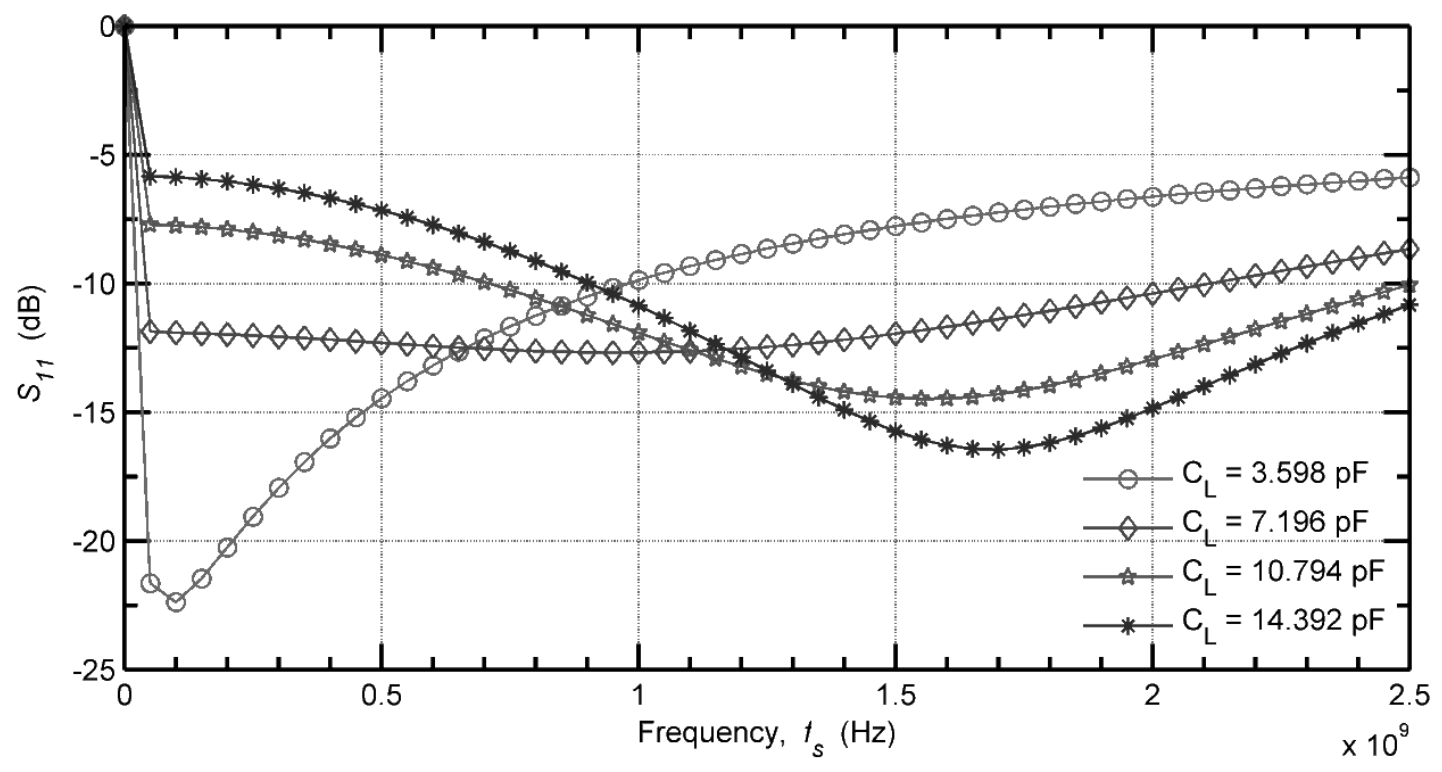

Figure 8 Determination of $C_{L}$ by parametric simulation of $S_{11}$ versus frequency.

For $C_{L}$ equal to $7.196 \mathrm{pF}, S_{11}$ is near-flat and is less than $-10 \mathrm{~dB}$ in a wide input frequency band of the LNA, from $300 \mathrm{MHz}$ to $1.4 \mathrm{GHz}$. For low values of $C_{L}$, the lower resonant frequency $f_{2}$ is dominant in $S_{11}$ response. For high values of $C_{L}, S_{11}$ tends to its minimum value, as shown in Figure 8 , and the frequency $f_{1}$ tends to $1.89 \mathrm{GHz}$. 


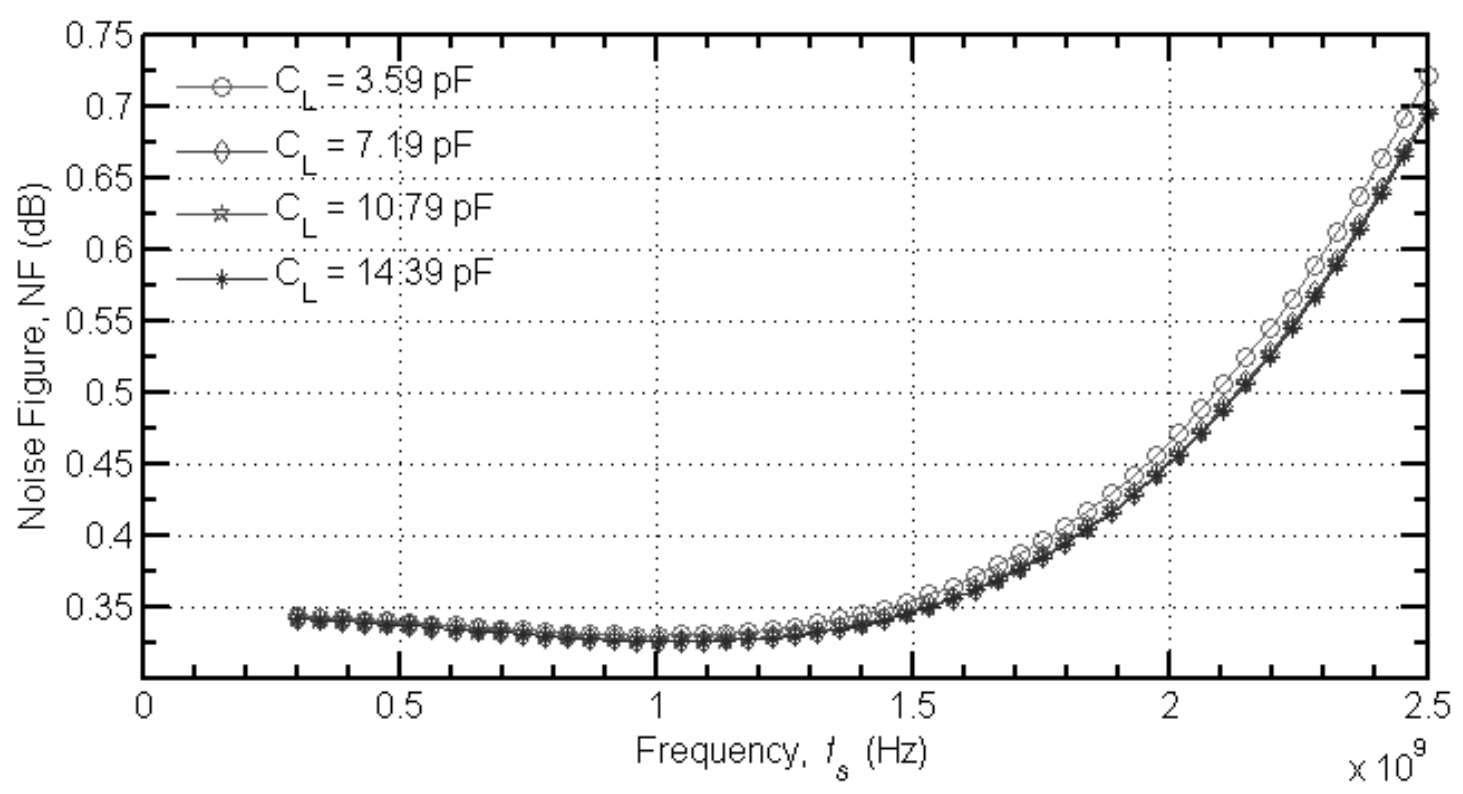

Figure 9 NF versus frequency and $C_{L .}$.

The plot of the NF shows in Figure 9 that the effect of $C_{L}$ on the NF is very small and that the NF varies slightly in the LNA bandwidth. At $1.4 \mathrm{GHz}$, the NF increases by about $0.002 \mathrm{~dB}$. However, for low values of $C_{L}$, the NF increases significantly.

\section{OUTPUT MATCHING}

The output impedance of the LNA is large, compared to the $50 \Omega$ of the load. In order to reduce NF impairments due to the matching network, a network with only reactive components is desired. However, a tentative preliminary design of the matching network done with the Smith chart shows that the trace of the reflection coefficient remains on the outer boundary of the chart; for the trace to move on circles of constant resistance on the $Z$ and $Y$ circles of the chart, a resistance $R_{L}$ is required at the output of the LNA, as shown in Figure 10. Although inductive peaking [17] is also a possible solution, it increases the operating voltage and significantly impairs the noise performance. 


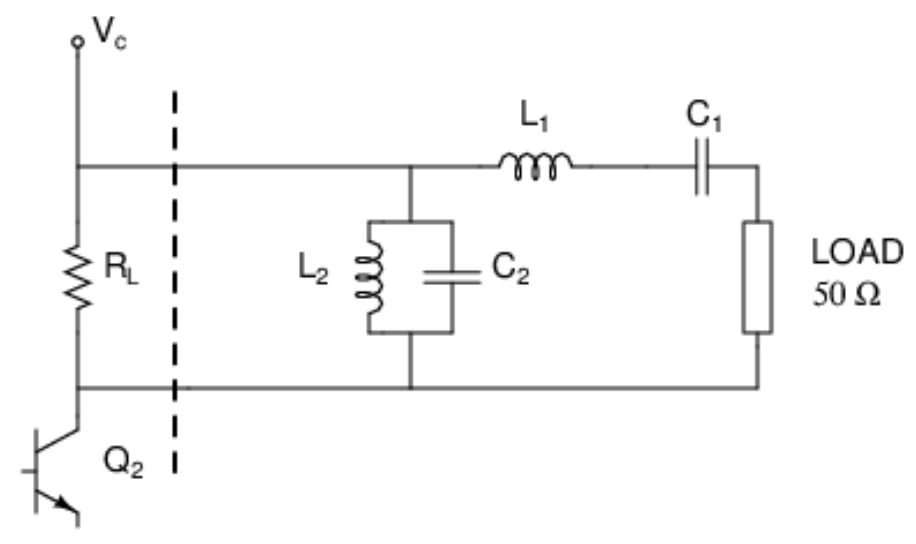

Figure 10 Output matching network.

A trade-off is required between NF and output matching: both the noise performance and the gain decrease with $R_{L}$ but the matching is impaired. For $R_{L}=90 \mathrm{ohms}$, it is found that broadband matching can be achieved by the network represented in Figure 10 , which may represent a $4^{\text {th }}$ order Butterworth or Chebyshev band-pass approximation, depending on the values of passive components. Although a passband filter can considerably reduce undesired signals, it does not maximize the transfer of power from its input to the output, unless the impedance of the input signal source is equal to the complex conjugate of the input impedance of the filter and the complex conjugate of the load impedance is equal to the output impedance of the filter. A MATLAB ${ }^{\mathrm{TM}}$ script is used to minimize the reflection of power resulting from a $4^{\text {th }}$ order filter based on a Butterworth approximation within the operating bandwidth. The script is based on the brute force technique that uses a computer-aided design tool to fine-tune the elements of a matching network based on a predetermined topology.

\section{FINAL LNA CIRCUIT}

The LNA circuit is represented in Figure 11, where the bias network is not shown. The base inductor $L_{B}$ and the choke are off-chip; all other components are on-chip. The size of SiGe HBTs used is $0.120 \mu \mathrm{m} \times 18 \mu \mathrm{m}$ with a multiplicity factor of 14 ; the supply voltage is $2.2 \mathrm{~V}$. 


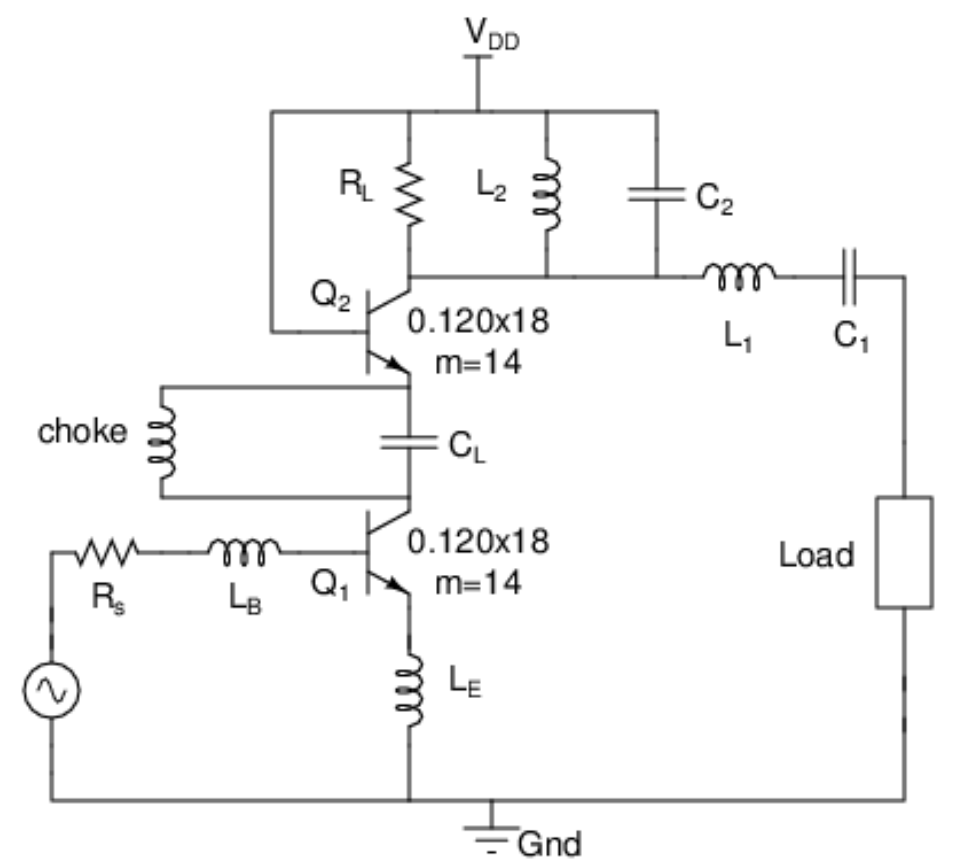

Figure 11 LNA circuit.

The resistance of the source is $R_{s}$, both $R_{s}$ and the resistance of the load are equal to $50 \Omega$. The plots of the NF, $S_{11}$ and $S_{22}$ are shown in Figure 12. The gain $G_{A}, S_{21}$ and $S_{12}$ are plotted in Figure 13 and Figure 14, respectively. The NF is less than $0.462 \mathrm{~dB}, S_{11}$ and $S_{22}$ are less than $10 \mathrm{~dB}$ from $300 \mathrm{MHz}$ to $1.4 \mathrm{GHz}$. All components are from the $130 \mathrm{~nm}$ IBM BiCMOS8HP process and correspond to eight layers of metal, except for the base inductor $L_{B}$ and the choke, which are external.

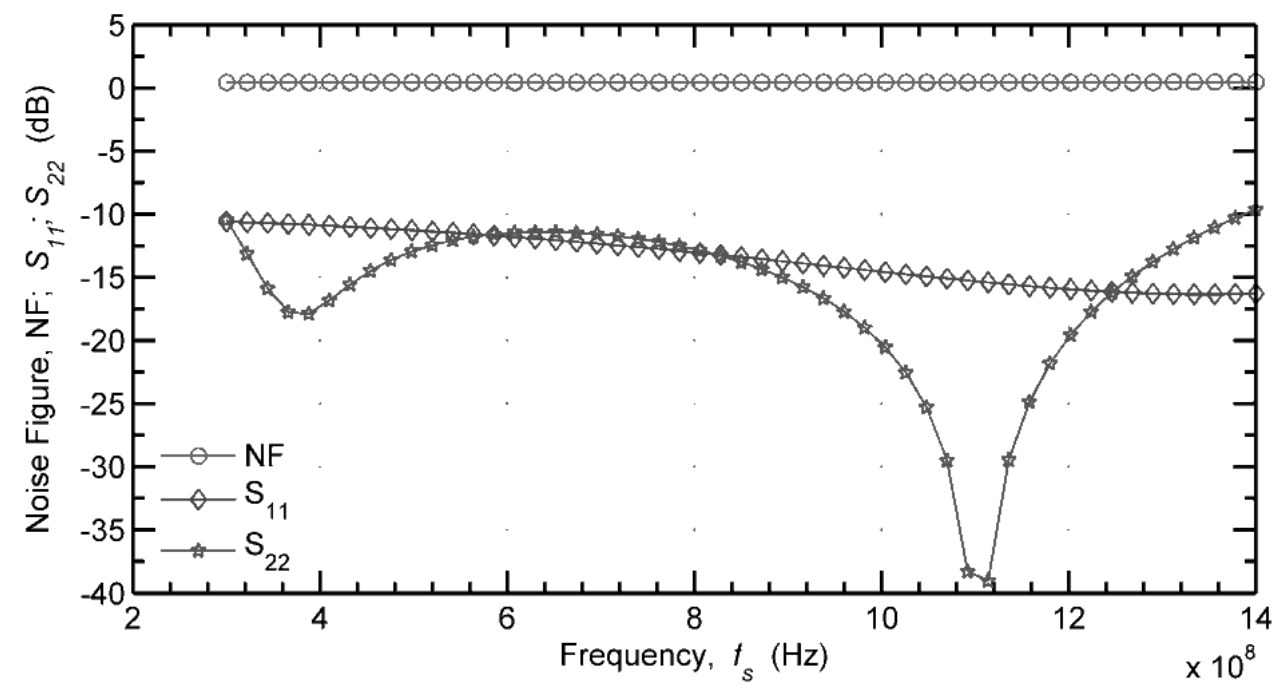

Figure 12 NF, $S_{11}$ and $S_{22}$ simulation by SpectreRF. 


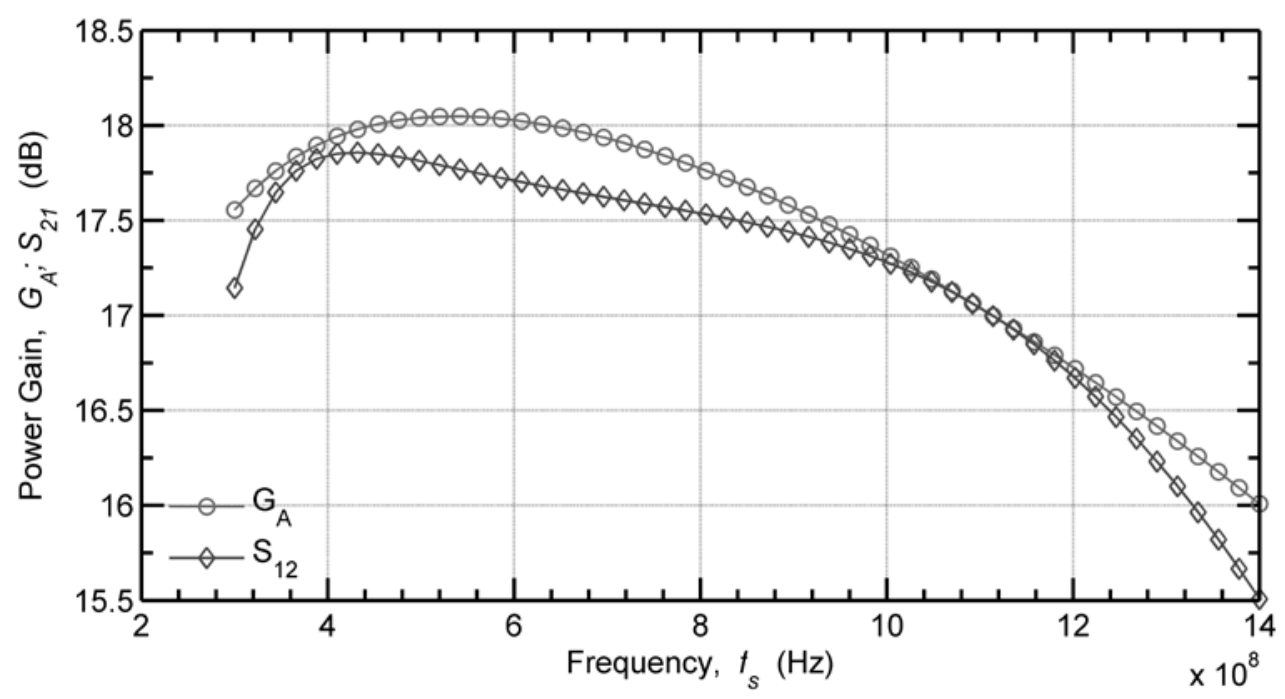

Figure $13 G_{A}$ and $S_{21}$ frequency response

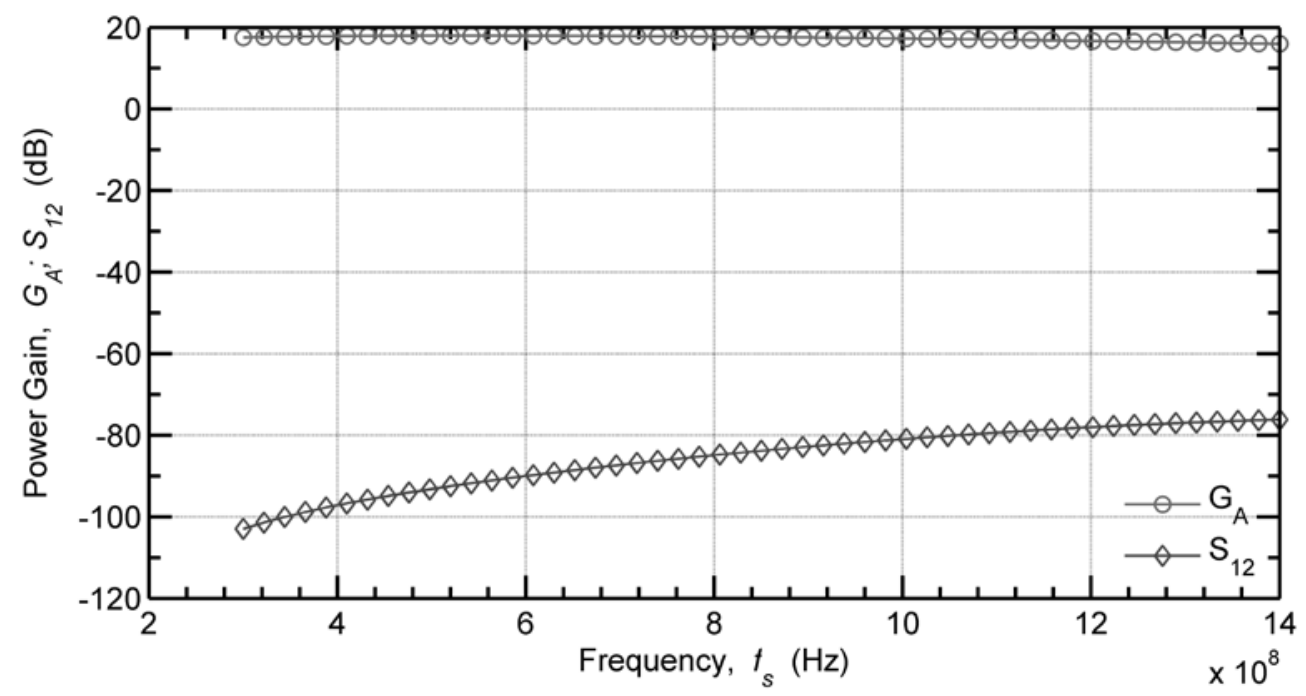

Figure $14 S_{12}$ frequency response.

The comparison between performance metrics of similar LNAs for radio astronomy and the impact of process, temperature, integration and bandwidth on performance is shown in Table 1. 
Table 1 Performance comparison with similar reported LNA designs.

\begin{tabular}{|c|c|c|c|c|c|c|}
\hline Property & [18] & [19] & [20] & [21] & [22] & $\begin{array}{c}\text { This work } \\
\text { (simulation) }\end{array}$ \\
\hline Technology $(\mu \mathrm{m})$ & $\begin{array}{l}0.090 \\
\text { CMOS }\end{array}$ & $\begin{array}{l}0.130 \text { IBM } \\
\text { BiCMOS8HP }\end{array}$ & $\begin{array}{l}\text { MMIC } 0.130 \text { IBM } \\
\text { BiCMOS8HP }\end{array}$ & $\begin{array}{l}\text { 1, InGaAs/InAlAs/ } \\
\text { Inp HEMT }\end{array}$ & $\begin{array}{l}\text { MIC Discrete } \\
\text { pHEMT }\end{array}$ & $\begin{array}{l}0.130 \text { IBM } \\
\text { BiCMOS8HP }\end{array}$ \\
\hline Frequency (GHz) & $0.7-1.4$ & $0.7-3$ & $0.1-5$ & $1-3$ & $0.02-1$ & $0.3-1.4$ \\
\hline $\mathrm{NF}(\mathrm{dB})$ & 0.35 & $<0.1$ & 1 & 0.4 & 0.55 & 0.46 \\
\hline $\mathrm{S}_{11}(\mathrm{~dB})$ & $<-11$ & $<0$ & $-8.4 @ 1.4 \mathrm{GHz}$ & $8.2 @ 1.4 \mathrm{GHz}$ & $<-4.5$ & $<-10$ \\
\hline $\mathrm{S}_{21}(\mathrm{~dB})$ & $20.5-16.3$ & 32.8 & $28.3 @ 1.4 \mathrm{GHz}$ & -16.5@1.4 GHz & $>36$ & $17.8 \max$ \\
\hline $\mathrm{S}_{22}(\mathrm{~dB})$ & $<-8$ & $<-12$ & $-16 @ 1.4 \mathrm{GHz}$ & -12.5 & $<-10$ & $<-10$ \\
\hline Gain (dB) & 17 & 28 & 27 & 30 & 30 & 18 \\
\hline Supply (V) & 1 & 0.6 & - & 1.8 & 1 & 2.2 \\
\hline Supply (mA) & 45 & 7.1 & - & 47 & 25 & 6 \\
\hline $\begin{array}{l}\text { Source resistance } \\
(\Omega)\end{array}$ & 50 & 50 & 50 & 50 & 50 & 50 \\
\hline $\begin{array}{l}\text { Single- } \\
\text { Ended/Balanced }\end{array}$ & S.E. & S.E. & S.E. & S.E. & S.E. & S.E. \\
\hline Temperature (K) & Room & Cryogenic (15) & Room & Room & Room & Room \\
\hline Area $\left(\mathrm{mm}^{2}\right)$ & 0.81 & - & - & $\begin{array}{l}\text { Results from } \\
\text { simulation }\end{array}$ & - & 1.4 \\
\hline External coils & 2 & 3 & 5 bond wires & 0 & - & 2 \\
\hline Publication year & 2007 & 2007 & 2009 & 2010 & 2014 & 2016 \\
\hline
\end{tabular}

\section{CONCLUSION}

A novel bandwidth expansion technique with minimum impact on NF has been proposed for integrated narrowband cascode SiGe HBT LNAs. This technique achieves wideband impedance matching at the input of the LNA by reducing to the absolute minimum the number of passive components and by using the capacitance of the reverse biased base-collector junction of the input transistor as network element. Input impedance matching is based on the coupling of two resonant circuits, resulting in the sharing of the energy of each tuned circuit by the other. Noise 
matching is done by varying the length of the emitter of transistors or by shunting several identical transistors.

The technique is applied to a sub-1 dB narrowband SiGe HBT LNA. It can be shown that the bandwidth of the narrowband LNA can be expanded more than five times, from $177 \mathrm{MHz}$ to more than $1.1 \mathrm{GHz}$, for applications in radio astronomy for the SKA. The impact on the NF is noticeably small and simulation by SpectreRF shows that the NF increases from $0.337 \mathrm{~dB}$ to $0.462 \mathrm{~dB}$ at room temperature. The maximum gain of the LNA is close to $18 \mathrm{~dB}, S_{11}$ and $S_{22}$ are less than $-10 \mathrm{~dB}$ in the bandwidth of the LNA.

Simulation has shown that the increase in the NF is mainly due to losses in the inductors of the output matching network. The base inductor $L_{B}$ and the choke are off-chip, the emitter inductor $L_{E}$ and inductors of the output matching network are on-chip. The noise performance of the LNA can be improved when inductors with a high quality factor are used.

\section{REFERENCES}

[1] A. Bimana and S. Sinha, "Impact of SiGe parameters to the performance of LNAs for highly sensitive SKA receivers." In Radioelektronika (RADIOELEKTRONIKA), $201323^{\text {rd }}$ International Conference, pp. 50-54. IEEE, 2013.

[2] D.M.P. Smith, L. Bakker, R.H. Witvers, B.E.M. Woestenburg and K.D. Palmer, "Low noise amplifier for radio astronomy," Int J.Microw. Wirel. Technol., vol. 5, no. 04, pp. 453-461, August 2013.

[3] E. Ojefors, F. Pourchon, P. Chevalier and U.R. Pfeiffer, "A 160-GHz low-noise downconversion receiver front-end in a SiGe HBT technology,” Int J.Microw. Wirel. Technol., vol. 3 no. 03, pp. 347-353, June 2011.

[4] H.A. Haus, W.R. Atkinson, G.M. Branch, W.B. Davenport, W.H. Fonger, W.A. Harris, S.W. Harrison, W.W. McLeod, E.K. Stodola and T.E. Talpey, "Representation of noise in linear twoports," Proc. IRE, vol. 48, no. 1, pp. 69-74, 1960.

[5] G. Vasilescu, Electronic Noise and Interfering Signals: Principles and Applications. Springer-Verlag, Heidelberg, Germany, 2005. 
[6] E.H. Nordholdt, Design of High-Performance Negative Feedback Amplifiers. Elsevier Scientific, Amsterdam, 1983.

[7] S.P. Voinigescu, M.C. Maliepaard, J.L. Showell, G.E. Babcock, D. Marchesan, M. Schroter, P. Schvan and D. Harame, “A scalable high-frequency noise model for bipolar transistors with application to optimal transistor sizing for low-noise amplifier design,” IEEE J. Solid-State Circuits, vol. 32, no. 9, pp. 1430-1438, 1997.

[8] L.N. Tran, "Caractérisation d'interconnexions et d'inductances en technologie BiCMOS. Application à l'amplification faible bruit,” Ph.D. dissertation, University of Cergy-Pontoise, Paris, France, May 2009.

[9] S. Voinigescu, High-Frequency Integrated Circuits. Cambridge Univ. Press, New York, 2013.

[10] H.T. Friis, “Noise figure of radio receivers,” Proc. IRE, vol. 32, no. 7, pp. 419-422, July 1944.

[11] D.M.P. Smith and B.E.M. Woestenburg, "Technique for reduction of noise resistance in a balanced low-noise amplifier for beam-steering applications,” Int J.Microw. Wirel. Technol., vol. 5, no. 05, pp. 561-565, October 2013.

[12] A. Bevilacqua and A.M. Niknejad, "An ultrawideband CMOS low noise amplifier for 3.110.6 GHz wireless receivers,” IEEE J. Solid-State Circuits, vol. 39, no. 12, pp. 2259-2268, December 2004.

[13] R. Hu and M.S.C. Yang, "Investigation of different input-matching mechanisms used in wide-band LNA design,” Int. J. Infrared Millimeter Waves, vol. 26, no. 2, pp. 221-245, February 2005.

[14] A.M. Niknejad, Electromagnetics for High-Speed Analog and Digital Communication Circuits. Cambridge Univ. Press, 2007.

[15] T. Lee, Planar Microwave Engineering: A Practical Guide to Theory, Measurement and Circuits. Cambridge Univ. Press, 2004. 
[16] N. Wadefalk, A. Mellberg, I. Angelov, M. Barsky, S. Bui, E. Choumas, R. Grundbacher, E. Kollberg, R. Lai, N. Rorsman, P. Starski, D. Stenarson, J. Streit and H. Zirath, "Cryogenic wideband ultra-low-noise IF amplifiers operating at ultra-low DC power," IEEE Trans. Microwave Theory Tech., vol. 51, no. 6, pp. 1705-1711, June 2003.

[17] M.T. Hsu, S.Y. Hsu and Y.H. Lin, "Low-power CMOS LNA based on dual resistivefeedback structure with peaking inductor for wideband application," Int J.Microw. Wirel. Technol, vol. 5 no. 01, pp. 65-70, February 2013.

[18] L. Belostotski and W. Haslett, "Wide band room temperature 0.35-dB noise figure LNA in 90-nm bulk CMOS,” in Proc. IEEE Radio Wireless Symp., Long Beach, CA, Jan. 7-11, 2007, pp. 221-224.

[19] S. Weinreb, J.C. Bardin and H. Mani, “Design of cryogenic SiGe low-noise amplifiers,” IEEE Trans. Microwave Theory Tech., vol. 55, pp. 2306-2312, November 2007.

[20] S. Weinreb, J.C. Bardin and H. Mani, “A 0.15-5 GHz cryogenic SiGe MMIC LNA,” IEEE Microwave and Wireless Comp. Lett., vol. 19, no. 6, pp. 407-409, June 2009.

[21] Z. Hamaizia, N. Sengouga, M. Missous and M.C.E. Yagoub, “A 0.4 dB noise figure wideband low-noise amplifier using a novel InGAs/InAlAs/InP device,” Materials Science in Semiconductor Processing, vol. 14, no. 2, pp. 89-93, June 2011.

[22] M. Panahi, S. Bhaumik and D. George, "Power-efficient ultra wideband LNAs for the world’s largest radio telescope,” Experimental Astronomy, vol. 38, no. 3, pp. 359-379, December 2014. 